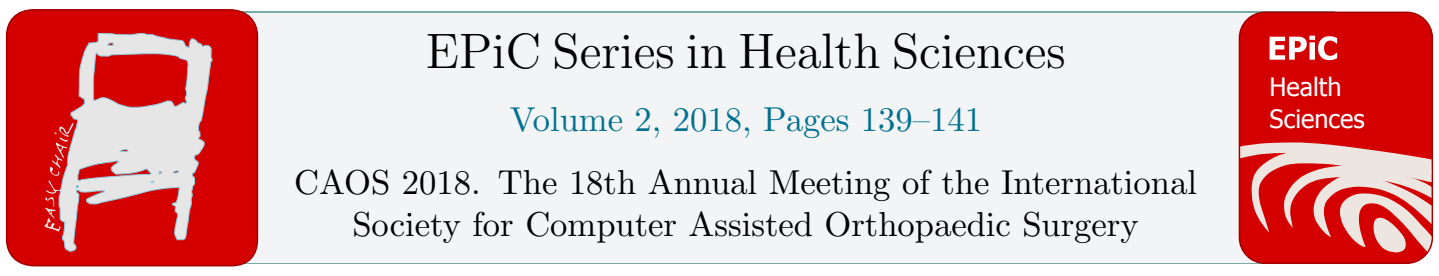

\title{
Accuracy of Mechanical Cup Alignment Device based on Functional Pelvic Plane in DAA-THA with Supine Position
}

\author{
Satoshi Nakasone ${ }^{1}$, Masato Ishihara ${ }^{1}$, Hiroyuki Taira ${ }^{1}$, Mika Takaesu $^{2}$, \\ Shuko Chinen ${ }^{2}$, Yoshihide Miyata ${ }^{2}$, Fuminori Kanaya ${ }^{1}$ \\ ${ }^{1}$ Department of Orthopedic Surgery, Graduate School of Medicine, University of the Ryukyus \\ ${ }^{2}$ Department of Orthopedic Surgery, Chubu-Tokushukai Hospital \\ s-nakasone@umin.ac.jp, satoshi_nakasone@hotmail.com
}

\begin{abstract}
Pelvic rotation during cup impaction causes excessive cup anteversion should be considered in direct anterior approach or anterior lateral approach total hip arthroplasty (THA) with supine position. There are two intraoperative support mechanical devices for THAs in supine position, one is based on functional pelvic plane (FPP) and the other is based on anatomical pelvic plane (APP). The purpose of this study was to compare the accuracy of two mechanical devices based on FPP or APP in THA with supine position.

The subjects of this study were 90 hips of 80 patients who underwent DAA-THA in a supine position using HipPointer ${ }^{\circledR}$ based on FPP (Pointer group). The other subjects of this study were 29 hips of 27 patients using HipCOMPASS ${ }^{\circledR}$ (Lexi, Tokyo) based on APP (COMPASS group). There are no significant differences in age, gender and BMI between the two groups.

Cup inclination and anteversion angle in Pointer group and COMPASS group were $40.2^{\circ}\left(33-48^{\circ}\right), 40.7^{\circ}\left(33-47^{\circ}\right)$, and $16.5^{\circ}\left(9-26^{\circ}\right), 17.5^{\circ}\left(10-27^{\circ}\right)$, respectively. There are no significant differences in cup inclination and anteversion angle between Pointer group and COMPASS group. Nor were there any significant differences in the absolute value errors for cup inclination and anteversion angle (inclination angle; $2.5^{\circ} \pm 2.1 \mathrm{vs} 2.4^{\circ}$

$\pm 2.0 、 \mathrm{P}=0.45$ ) (Anteversion; $2.7^{\circ} \pm 2.4$ vs $3.0^{\circ} \pm 2.0 、 \mathrm{P}=0.23$ ).

New developed mechanical navigation device based on intraoperative FPP by correction of axially rotated pelvis to surgical bed plane in the DAA-THA is an simple and useful device which accuracy of cup alignment didn't show significant differences compared with the device based on intraoperative APP with reference of pubic symphysis.
\end{abstract}




\section{Introduction}

Direct anterior approach (DAA-THA) in performing the total hip arthroplasty could stabilize hip joint better than lateral decubitus position and higher accuracy of cup alignment setting has been reported than that of THA in decubitus position ${ }^{1)}$. However, excessive anteversion of the cup in DAATHA have been reported by the pelvic rotation to the operative side by traction of acetabular retractors and the interference of cup impactor by the femur ${ }^{2)}$. We need to consider pelvic rotation for correct setting the acetabular cup. There are commercially available mechanical devices which based on anatomical pelvic plane (APP) referring to bilateral anterior superior iliac spine (ASIS) and pubic symphysis. We reported the cup alignment accuracy with use of this device based on APP before ${ }^{3)}$. However, exact setting of devices was difficult in some cases because of thick distance from skin to pubic symphysis. Therefore, we developed a new cup alignment device based on functional pelvic plane (FPP) referring to surgical bed plane through bilateral ASIS during surgery which can correct pelvic rotation. The purpose of this study was to compare the accuracy of two mechanical cup alignment devices.

\section{Patients and methods}

The subjects of this study were 90 hips of 80 patients who underwent DAA-THA in a supine position using HipPointer ${ }^{\circledR}$ based on FPP from July 2016 to December 2017 (Pointer group). Pointer group consisted of 31 males (37 hips) and 49 females (53 hips). The other subjects of this study were 29 hips of 27 patients using HipCOMPASS ${ }^{\circledR}$ (Lexi, Tokyo) based on APP from April 2013 to May 2014 (COMPASS group). COMPASS group consisted of 10 males (11hips) and 17 females (18hips). There are no significant differences in age, gender and BMI between the two groups.

In both groups, three-dimensional CT based preoperative plan was performed by software (ZedHip ${ }^{\circledR}$, Tokyo, Japan) on FPP, where the pelvis in supine position on the CT scan table that was axially rotated until the bilateral ASIS touched the same CT table plane, with radiographic definition with an inclination of $40^{\circ}$ and $15^{\circ}$ of anteversion principally.

HipPointer ${ }^{\circledR}$ consist of the body, two adjustable arms and indicator, which was fixed to the bilateral ASIS with inserted $2.0 \mathrm{~mm} \mathrm{~K}$-wire (Fig. 1). Therefore HipPointer ${ }^{\circledR}$ is flexible fixation in pelvic sagittal plane. To create FPP during surgery, pelvic axially rotation corrected parallel to surgical bed plane by pressing bilateral ASIS through arms of the device. Then the cup was impacted with the insertion handle aligned along with the indicator (Fig. 2). On the other hand, HipCOMPASS ${ }^{\circledR}$ consists of the body, adjustable three arms and indicator, which can input the values by ZedHip ${ }^{\circledR}$ automatically calculated length of bilateral ASIS, perpendicular length from the pubic symphysis and cups alignment with radiographic definition as an indicator of inclination and anteversion (Fig. 3). To
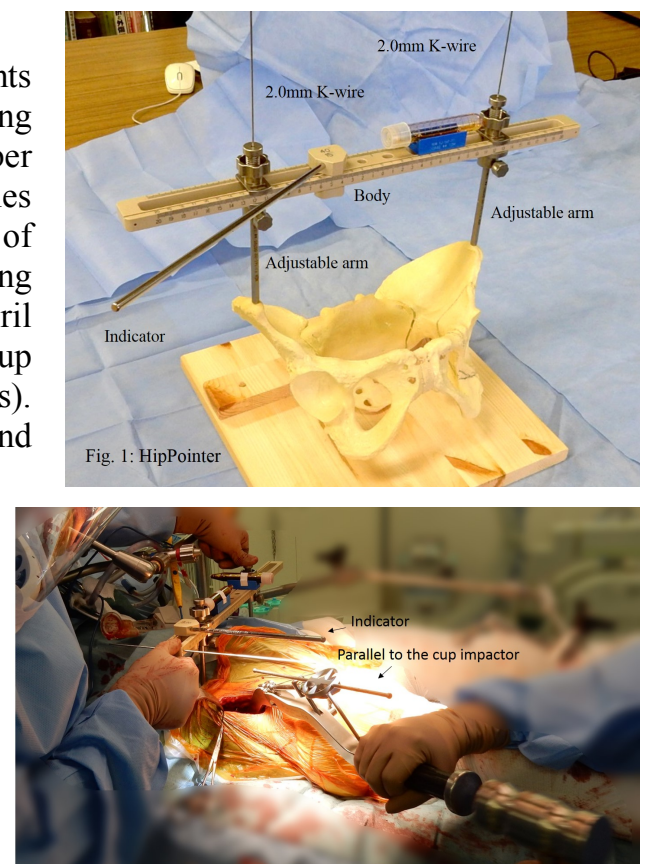

Fig. 2: In use of a HipPointer, cup impaction aligned parallel to the indicator

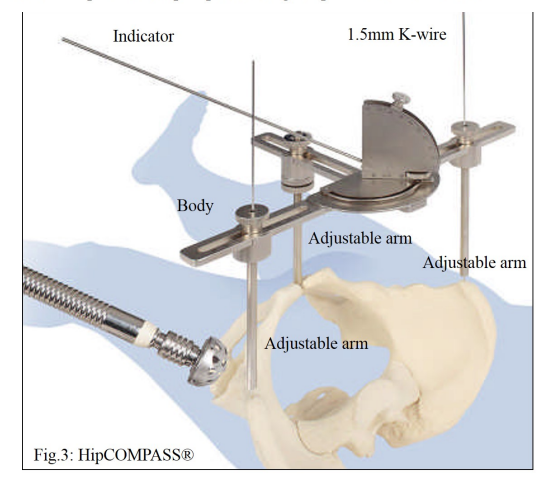


create APP during surgery, HipCOMPASS ${ }^{\circledR}$ was fixed to the bilateral ASIS and pubic symphysis. Then the cup was placed along indicator based on APP.

Cup alignment was converted to radiographic definition using 3-D preoperative plan and postoperative CT data by postoperative ZedHip ${ }^{\circledR}$ (Tokyo, Japan). Accuracy of cup alignment was compared absolute values between both groups.

\section{Results}

Cup inclination and anteversion angle in Pointer group and COMPASS group were $40.2^{\circ}\left(33-48^{\circ}\right)$, $40.7^{\circ}\left(33-47^{\circ}\right)$, and $16.5^{\circ}\left(9-26^{\circ}\right), 17.5^{\circ}\left(10-27^{\circ}\right)$, respectively. There are no significant differences in cup inclination and anteversion angle between Pointer group and COMPASS group. Nor were there any significant differences in the absolute value errors for cup inclination and anteversion angle (inclination angle; $2.5^{\circ} \pm 2.1$ vs $2.4^{\circ} \pm 2.0 、 \mathrm{P}=0.45$ ) (Anteversion; $2.7^{\circ} \pm 2.4$ vs $3.0^{\circ} \pm 2.0 、 \mathrm{P}=0.23$ ).

\section{Discussion}

Accuracy of cup alignment with use of a new developed mechanical navigation device based on intraoperative FPP by correction of axially rotated pelvis to the surgical bed plane in the DAA-THA didn't show significant difference compared with use of a HipCOMPASS ${ }^{\circledR}$ based on intraoperative $\mathrm{APP}^{3)}$., that of mechanical navigation ${ }^{4)}$ and computer navigation ${ }^{5)}$ as well. Although HipPointer ${ }^{\circledR}$ cannot correct the pelvic sagittal rotation during surgery, the absolute value errors are only $2.5^{\circ}$ cup inclination and $2.7^{\circ}$ anteversion angle, and so such limitation is not expected to cause great errors in the accurate of cup alignment.

The surgeon and assistant can visually confirm the rotated pelvis corrected by HipPointer® during surgery, the surgeon can impact the cup accurately as usual, though the mechanical device based on APP which referring to various pelvic axial and sagittal rotation by each patient indicates various direction for cup alignment during surgery. Thus, this mechanical navigation device based on intraoperative FPP is a simple and useful device that can set the cup alignment accurate in DAA-THA compared with the device based on APP with reference of pubic symphysis.

\section{References}

1) Higgins, BT. et al.: Anterior vs. Posterior Approach for Total Hip Arthroplasty, a Systematic Review and Meta-analysis, J Arthroplasty. 419-434, 2015.

2) Kobayashi, H. et al.; Surgeons changing the approach for total hip arthroplasty from posterior to direct anterior with fluoroscopy should consider potential excessive cup anteversion and flexion implantation of the stem in their early experience. Int Orthop., 2015.

3) Accuracy of mechanical cup alignment device HipCOMPASS in DAA-THA with supine position.: Hip Joint(40), 816-820. 2014.

4) Steppacher, SD. et al.: Improving cup positioning using a mechanical navigation instrument. Clin Orthop Relat Res. 469, 423-8. 2011.

5) Sugano, N. et al. Does CT-Based Navigation Improve the Long-Term Survival in Ceramic-onCeramic THA? Clin Orthop Relat Res. 2012. 